\title{
Multispacecraft observations of a prominence eruption
}

\author{
A. Bemporad ${ }^{1}$, G. Del Zanna ${ }^{2}$, V. Andretta ${ }^{3}$, G. Poletto ${ }^{4}$, and M. Magrí ${ }^{3}$ \\ ${ }^{1}$ INAF - Torino Astronomical Obs., via Osservatorio 20, 10025 Pino Torinese (TO), Italy \\ ${ }^{2}$ Dept. of Applied Mathematics and Theoretical Physics, Univ. of Cambridge, Wilberforce Road, Cambridge CB3 OWA, UK \\ ${ }^{3}$ INAF - Capodimonte Astronomical Obs., salita Moiariello 16, 80131 Napoli, Italy \\ ${ }^{4}$ INAF - Arcetri Astrophysical Obs., L.go E. Fermi 5, 50125 Firenze, Italy
}

Received: 3 June 2009 - Revised: 24 August 2009 - Accepted: 1 September 2009 - Published: 9 October 2009

\begin{abstract}
On 9 May 2007 a prominence eruption occurred at the West limb. Remarkably, the event was observed by the STEREO/EUVI telescopes and by the HINODE/EIS and SOHO/UVCS spectrometers. We present results from all these instruments. High-cadence $(\sim 37 \mathrm{~s})$ data from STEREO/EUVI A and B in the He II $\lambda 304$ line were used to study the 3-D shape and expansion of the prominence. The high spatial resolution EUVI images $\left(\sim 1.5^{\prime \prime} /\right.$ pixel) have been used to infer via triangulation the 3-D shape and orientation of the prominence $12 \mathrm{~min}$ after the eruption onset. At this time the prominence has mainly the shape of a "hook" highly inclined southward, has an average thickness of $0.068 \mathrm{R}_{\odot}$, a length of $0.43 \mathrm{R}_{\odot}$ and lies, in first approximation, on a plane. Hence, the prominence is mainly a 2-D structure and there is no evidence for a twisted flux rope configuration. HINODE/EIS was scanning with the $2^{\prime \prime}$ slit the region where the filament erupted. The EIS spectra show during the eruption remarkable non-thermal broadening (up to $\sim 100 \mathrm{~km} \mathrm{~s}^{-1}$ ) in the region crossed by the filament in spectral lines emitted at different temperatures, possibly with differences among lines from higher Fe ionization stages. The CME was also observed by the SOHO/UVCS instrument: the spectrograph slit was centered at $1.7 \mathrm{R}_{\odot}$, at a latitude of $5^{\circ} \mathrm{SW}$ and recorded a sudden increase in the O VI $\lambda \lambda 1032-1037$ and Si XII $\lambda 520$ spectral line intensities, representative of the CME front transit.
\end{abstract}

Keywords. Solar physics, astrophysics, and astronomy (Flares and mass ejections; Ultraviolet emissions) - Space plasma physics (Magnetic reconnection)

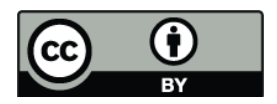

Correspondence to: A. Bemporad (bemporad@oato.inaf.it)

\section{Introduction}

Despite the huge number of solar coronal mass ejections (CMEs) studied in the last decades from coronagraphic and spectroscopic data, many fundamental questions on these events are still open. In particular, even if it is at present clear that CMEs are related to the storage and subsequent release of magnetic energy in the chromosphere and the lower corona, the real physical phenomena triggering the final energy release have not yet been unambiguously identified. Due to the intrinsic complexity of solar eruptions, it is necessary to observe these events with as many instruments as possible covering the whole electromagnetic spectrum, to have information on particle accelerations and shocks (from radio data), on coronal densities (from coronagraphic white light images), coronal temperatures (from EUV telescopes), kinetic temperatures and plasma motions (from EUV spectroscopic data), and energy deposition sites (from X-ray data). Hence it is mandatory to focus on the events occurring during multi-spacecraft campaigns.

In May 2007 we designed a multi-spacecraft campaign aimed at observing the off-limb corona with Hinode, STEREO and SOHO instruments (Hinode HOP 7; see Del Zanna et al., 2009) and at measuring electron temperatures, densities, and elemental abundances in the low corona at different altitudes and latitudes above an Active Region (AR - NOAA 10953). This campaign involved in particular the STEREO EUV Imager (EUVI; see Wuelser et al., 2004), the HINODE EUV Imaging Spectrometer (EIS; see Culhane et al., 2007) and the SOHO Ultraviolet Coronagraph Spectrometer (UVCS; see Kohl et al., 1995). During this campaign, a prominence eruption and a coronal mass ejection (CME) occurred on May 9 at the West limb (see also Bemporad et al., 2009a). This unique event has been observed by all the instruments mentioned above: in the following we present the results we obtained from imaging data acquired by STEREO/EUVI (Sect. 2), which has been used to make

Published by Copernicus Publications on behalf of the European Geosciences Union. 

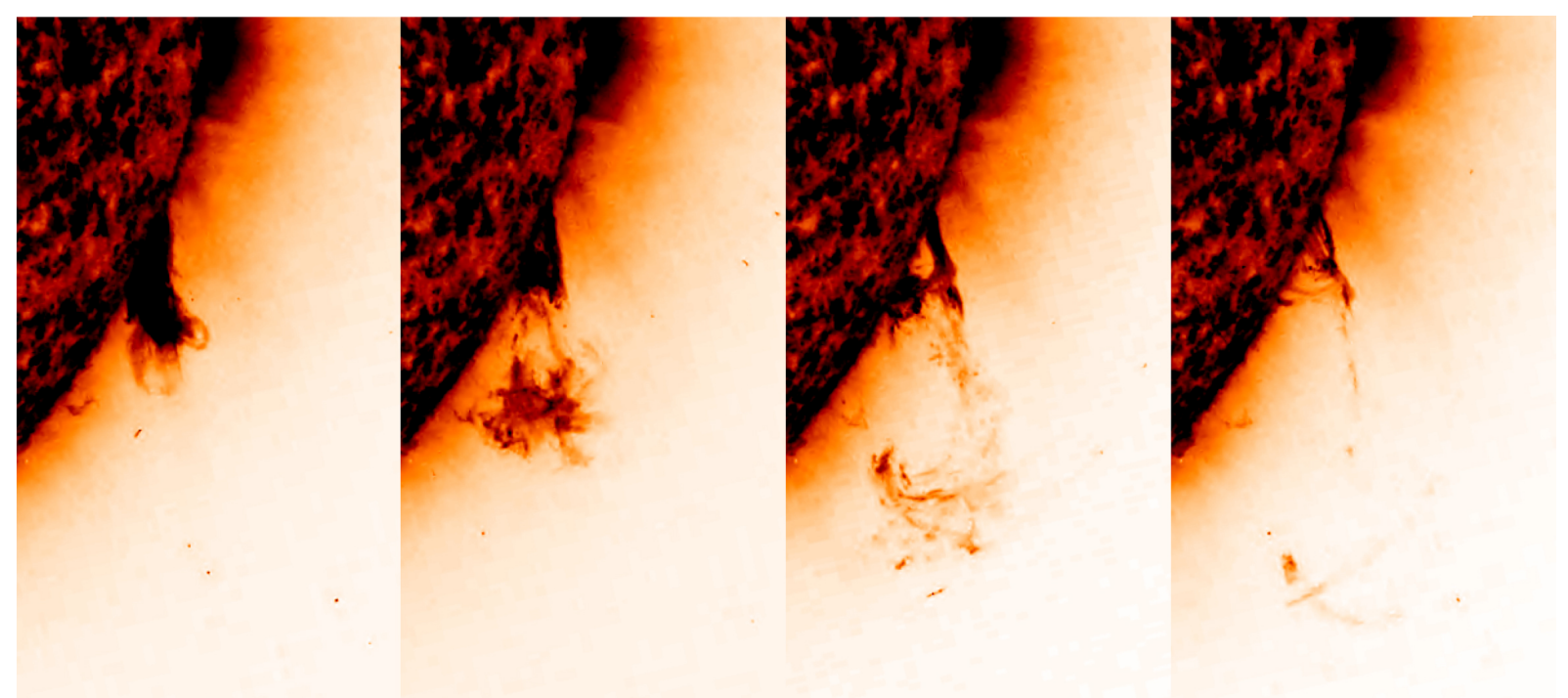

STEREO B/EUVI, 13:40 STEREO B/EUVI, 13:46 STEREO B/EUVI, 13:52 STEREO B/EUVI, 13:58

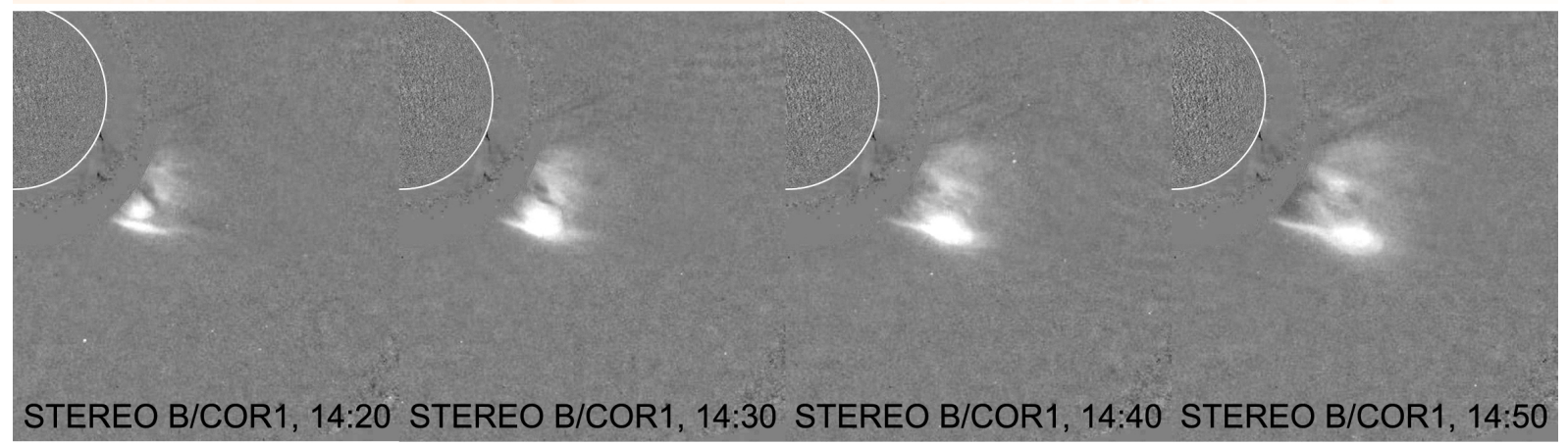

Fig. 1. Top: sequence of He II $\lambda 304$ images acquired by the EUVI telescope on the STEREO-B spacecraft showing the erupting prominence object of the present study. Images are plotted in reverse color scale, hence darker features correspond to a brighter He II emission and viceversa. Bottom: the resulting coronal mass ejection as observed by the COR1 coronagraph aboard STEREO-B (sequence of base difference images; brighter features correspond to relative column density increases).

a 3-dimensional (3-D) reconstruction of the prominence, and from spectroscopic data acquired by HINODE/EIS (Sect. 3) and SOHO/UVCS (Sect. 4) instruments. Results are discussed and summarized in the last section (Sect. 5).

\section{STEREO/EUVI observations}

Between 25 April and 8 May 2007 the AR NOAA 10953, located at a latitude of $11^{\circ} \mathrm{S}$, crossed the disk dragged by the solar rotation. A H- $\alpha$ "S-shaped" filament was visible in these days above the source AR 10953, mostly aligned along the North-South direction: this filament is probably the source of the eruption on 9 May 2007. On this day, when the AR was already behind the solar limb by $\sim 14^{\circ}\left(11^{\circ} \mathrm{S}\right.$, $104^{\circ} \mathrm{W}$ ), a prominence eruption occurred (Fig. 1, top panels). In particular, the sequence of STEREO-B/EUVI He II $\lambda 304$ images shows a tongue of plasma being ejected start- ing from $\sim 13: 40 \mathrm{UT}$. The eruption emanates from a latitude of $\sim 27^{\circ} \mathrm{S}$, but in the early phase propagates southward (i.e. towards larger latitudes), highly inclined with respect to the radial direction (13:46 UT frame). Nevertheless, the following images show that (as it appears in the standard 2-D images, i.e. projected on the plane of the sky - see Fig. 1) the eruption changes its direction of propagation, rotating Westward and being finally ejected at a much higher latitude than the source AR. The prominence eruption was followed in the higher corona by a slow $\left(v_{\mathrm{CME}} \sim 310 \mathrm{~km} \mathrm{~s}^{-1}\right)$, decelerating $\left(a_{\mathrm{CME}} \sim-7.4 \mathrm{~m} \mathrm{~s}^{-2}\right)$ limb $\mathrm{CME}^{1}$ propagating around a latitude of $42^{\circ} \mathrm{S}$. The CME is shown in Fig. 1 (bottom panels) as a sequence of base difference STEREO-B/COR1 images (i.e. the last image before the CME has been subtracted from all following frames) representing the coronal density

\footnotetext{
${ }^{1}$ See the SOHO/LASCO CME catalog available on-line at http: //cdaw.gsfc.nasa.gov/CME_list/.
} 

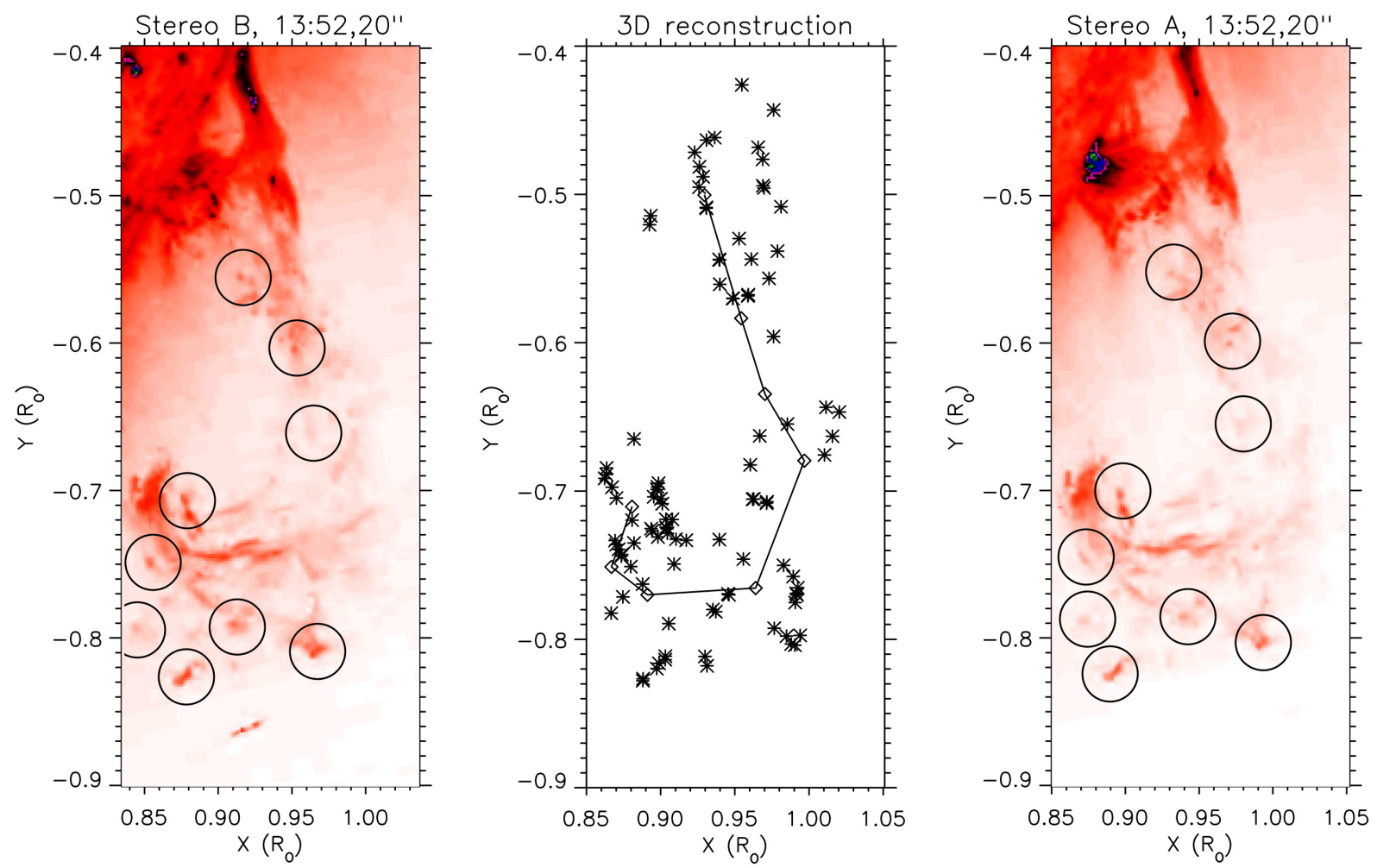

Fig. 2. The pair of He II $\lambda 304$ images acquired at 13:52 UT by EUVI aboard STEREO B (left panel) and A (right panel) and the 3-D positions (reconstructed via triangulation) of the 100 pairs of features selected inside the prominence as seen from the Earth (middle panel). As an example, some of the selected pairs are outlined in left and right panels by white solid circles.

enhancements associated with the CME transit. Below the COR1 occulter the last EUVI image in the top row of Fig. 1 (13:58 UT) has been overplotted (base difference image) in order to better show the relative positioning of the prominence eruption and the resulting CME.

As mentioned in Sect. 1, these observations have been acquired during a multi-spacecraft campaign: in particular, on 7 May 2009, the STEREO/EUVI telescopes acquired a series of high cadence ( $\sim 37 \mathrm{~s})$ images in the He II $\lambda 304$ filter, showing the chromospheric plasma at temperatures around $\sim 6 \times 10^{4} \mathrm{~K}$. EUV images acquired by the two STEREO-A and -B spacecraft can be combined to perform a triangulation study and derive information on the 3-D shape of the erupting prominence. In order to perform this study it is necessary to identify the same features observed across the prominence in a pair of images acquired at the same time from two different points of view. On May 2007 the angle $\gamma$ between the twin STEREO spacecraft was quite small, around $\gamma \simeq 7.2^{\circ}$ : this helped us in the identification of the pair of features (such as plasma blobs, sub-filaments, etc...), even if on the other side a small angle between the two STEREO spacecraft implies larger uncertainties in the determination of the longitude $\lambda$ via the triangulation technique. This is a general result which can be demonstrated simply by differentiating the
Eqs. (1) given by Mierla et al. (2008): it turns out that, for constant uncertainties in all the measured parameters, the relative uncertainty $\Delta \lambda / \lambda$ on the longitude maximizes if $\gamma=0$ and progressively decreases as $\gamma$ increases.

In the first EUVI frames acquired during the eruption (see Fig. 1, 13:40 UT frame) it is difficult to identify many pairs of sub-features within the prominence to be used to perform the triangulation study, while at later times (see Fig. 1, 13:58 UT frame) the erupting plasma is no longer visible in the EUVI field of view (FOV). Fortunately, the high temporal cadence ( $\sim 37 \mathrm{~s})$ gave us the opportunity to select, among different pairs of STEREO images, the one showing the largest number of prominence features clearly identifiable at the same time from both points of view. Some of these pairs are outlined, as an example, in Fig. 2 (solid circles): in particular in this study we selected the pair of frames acquired at 13:52 UT. At this time the prominence is already well on its way, thus the prominence plasma is becoming increasingly "fragmented" and possibly diluted, making it possible to identify many relatively compact sub-features across the erupting material, a more difficult task at earlier and later times. Notice that this is also approximately the time when the EIS slit start to scan the erupting prominence (see later on, Fig. 4, top right panel). Once the pair association is made 


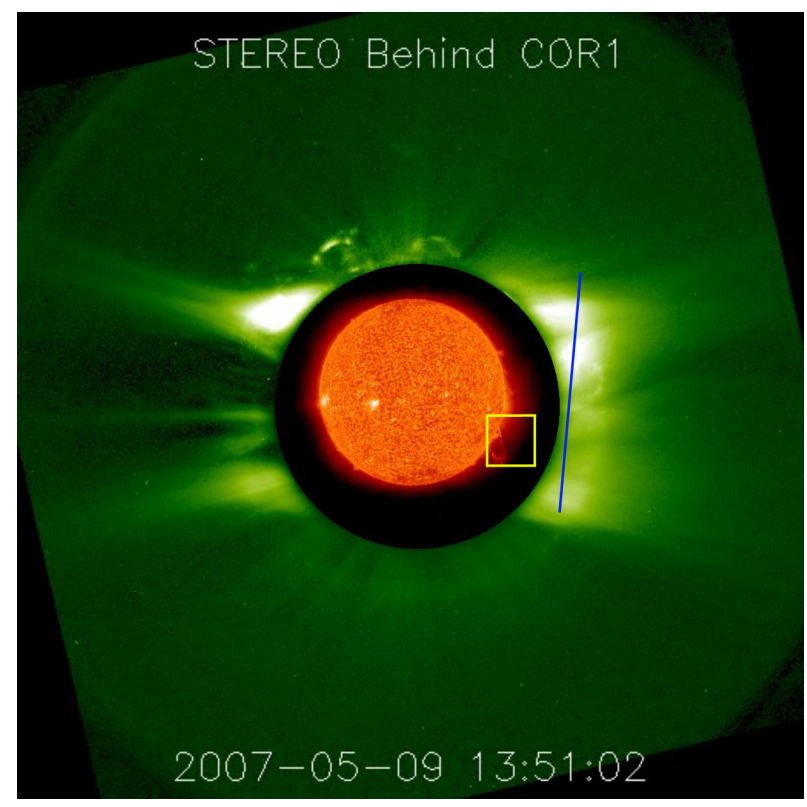

Fig. 3. STEREO-B COR 1 and EUVI He II $\lambda 304$ composite image acquired during the prominence eruption (13:51 UT) showing the field of views covered by the SOHO/UVCS slit (blue solid line) and by the HINODE/EIS spatial raster (yellow box).

(i.e. when the same source point $P$ is identified in both images), it is possible to derive the 3-D position of $P$ by using the geometrical relationships defined in the epipolar geometry. In particular, when $P$ is observed from two different points of view by the two STEREO-A and B telescopes, the same point is focused onto the two CCD detectors over two different image points $P_{A}$ and $P_{B}$; the three points $P, P_{A}$ and $P_{B}$ define the epipolar plane. If the positions of the two image points $P_{A}$ and $P_{B}$ (i.e. projected altitude and latitude) are known to correspond to the same 3-D point $P$ and if the position of the two spacecraft (in particular the angle $\gamma$ between the spacecraft) is known, then the 3-D position of $P$ can be calculated: this process is called triangulation (please refer to Mierla et al., 2008, for analytic formulas and for a better description of the STEREO triangulation geometry). By performing the same triangulation study over a large number of image point pairs located in the erupting prominence, the 3-D position and orientation of the erupting plasma can be reconstructed.

In this work we selected 100 pair of points across the prominence as observed by the two STEREO/EUVI telescopes at 13:52 UT: the resulting positions of these points are shown in the middle panel of Fig. 2 in the HeliocentricCartesian coordinate system. This reference system has the origin on the Sun center and the $\mathrm{x}-, \mathrm{y}-$ and $\mathrm{z}$-axes pointing towards the West limb, North limb (as seen from the Earth) and the Earth, respectively (see Fig. 2 in Thompson, 2006). By averaging the 3-D coordinates of these 100 points over
8 sub-classes of closest points, we derived 8 interpolating points. The solid line connecting these 8 average points (middle panel in Fig. 2) shows that at this time the prominence has approximately the shape of a "hook" anchored at the Sun surface. The "hook", oriented along the North-South direction, is not lying on the plane of the sky, but is inclined with respect to that plane by $\sim 35^{\circ}$ towards the observer. The prominence is centered at an average longitude of $14.2^{\circ}$ behind the limb (in very good agreement with the longitude of the source AR) and a latitude of $34.8^{\circ} \mathrm{S}$ (hence $\sim 24^{\circ}$ Southward with respect to the source AR). This implies that the prominence plasma motion, finally resulting in a small CME propagating at a latitude of $\sim 42^{\circ} \mathrm{S}$, occurs with negligible longitudinal velocity: the plasma expands mainly in altitude and in the latitudinal direction, while the longitudinal expansion rate is much smaller (please refer to Bemporad, 2009b, for a more detailed description of the results we derived on the 3-D prominence structure and expansion). The approximate prominence length (estimated as the sum of relative distances between the 8 average points) is $l=0.43 \mathrm{R}_{\odot}$, while the prominence thickness $d_{z}$ along the line of sight (LOS estimated as $d_{z}=2 \sigma_{z}$ where $\sigma_{z}$ is the average standard deviation of the z-coordinates for the 100 points) is $d_{z}=0.068 \mathrm{R}_{\odot}$; hence $l \sim 6.3 \times d_{z}$. As a consequence, we conclude that the prominence is mainly a 2-D structure and its material is expanding, at least in the early phase reported here, only along the latitudinal and radial directions, while negligible motions occurred in the longitudinal direction.

\section{HINODE/EIS observations}

During the multi-spacecraft campaign of May 2007, the HINODE/EIS spectrometer observed off limb in the SouthWest quadrant (yellow box in Fig. 3). The EIS instrument is able to observe spectral lines in two wavelength bands (SW: 163-209 $\AA$; LW: 242-289 $)$ ) with a spectral resolution of $0.0223 \AA /$ pixel and a spatial resolution of $1^{\prime \prime}$ along the slit; the instrument has 4 possible slit widths, two for spectroscopy ( 1 " and $\left.2^{\prime \prime}\right)$ and two for imaging (40" and $\left.266^{\prime \prime}\right)$ and acquires data with its 512" long slit oriented parallel to the North-South direction. On 9 May 2007 the instrument performed 5 spatial rasters: each raster, acquired over a time interval of about $1 \mathrm{~h}$ with the $2^{\prime \prime}$ slit, covered a FOV of $479.2 \times 512 \operatorname{arcsec}^{2}(\mathrm{X} \times \mathrm{Y})$, centered at the position $\mathrm{X}=985.2^{\prime \prime}, \mathrm{Y}=-491.7^{\prime \prime}$ in heliocentric coordinates (see Fig. 3). This FOV has been covered by moving the EIS $2^{\prime \prime}$ slit from West to East by steps of $7.8^{\prime \prime}$, covering a latitude interval between $10.9^{\circ}-45.1^{\circ} \mathrm{S}$ and an altitude interval between $0.81-1.49 \mathrm{R}_{\odot}$.

One of these rasters started at 13:15 UT and ended at 14:18 UT, hence covered the right time interval to observe the prominence eruption. Because the raster has been performed by moving the EIS slit from West to East, at the early phase of the eruption the slit was located at larger heliocentric 

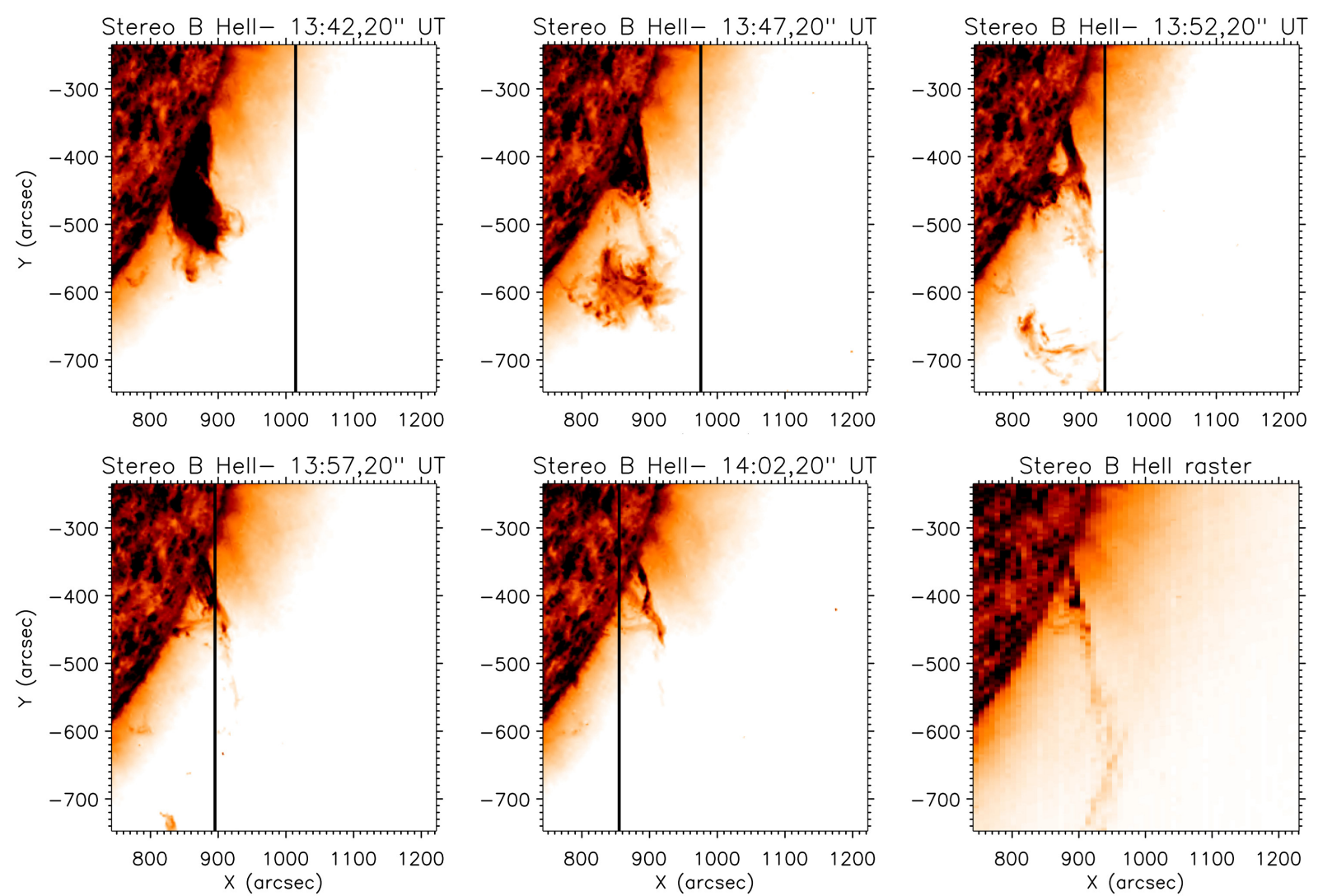

Fig. 4. Sequence of STEREO-B EUVI He II $\lambda 304$ images showing the evolution of chromospheric material in the HINODE/EIS field of view (yellow box in Fig. 3) during the $\sim 1 \mathrm{~h}$ raster acquired from 13:15 to 14:18 UT. The vertical black line shows the instantaneous position of the EIS slit during the West-East scanning. The bottom right panel shows the simulated raster resulting from STEREO/EUVI data to be compared directly with EIS line intensity maps (see Fig. 5).

distances, while the late phase of the eruption has been sampled at lower altitudes. In order to better compare the instantaneous FOV of the EIS slit with the chromospheric evolution observed by STEREO/EUVI in the He II $\lambda 304$ line, Fig. 4 shows a sequence of EUVI images in the FOV covered by the whole EIS raster (see above): the vertical black line represents the instantaneous position of the EIS slit during the raster. This sequence demonstrates that the EIS slit cut exactly across the prominence during its eruption. For future comparison with EIS intensity maps, the bottom right panel of Fig. 4 shows also the He II $\lambda 304$ intensity map constructed by cutting vertical slices from STEREO/EUVI images at positions and times of the EIS slit FOV and by degrading the spatial resolution in the $\mathrm{X}$ direction down to the EIS resolution. Hence, this panel is a simulated "STEREO/EUVI raster" and shows what we expect to observe in EIS data, for instance in the He II $\lambda 256$ line intensity map. In particular, the simulated EIS raster (bottom right panel of Fig. 4) shows something like a vertical filament of chromospheric material. As demonstrated by this figure, this is the net result of the superposition between two simultaneous movements: the Westward expansion of the erupting prominence and the Eastward scan of the EIS slit making the spatial raster.

The EIS intensity maps (Fig. 5) in different lines, obtained by integrating over each line profile, show enhanced emission of the chromospheric erupting material only in the "cooler" spectral lines such as He II $\lambda 256.32$ (top left panel) and Fe VIII $\lambda 185.21$ (top right); these two lines have maximum formation temperatures of $T \sim 10^{4.9} \mathrm{~K}$ and $T \sim 10^{5.6} \mathrm{~K}$, respectively. The He II $\lambda 256.32$ intensity map ${ }^{2}$ from EIS is in good agreement with the map derived from STEREO/EUVI data in $\mathrm{He}$ II $\lambda 304$, as expected. A comparison between Fig. 5 and Fig. 4 shows that the observed EIS He II intensity map is a complex superposition of the spatial and temporal evolutions occurring during the eruption. At $\sim 13: 50$ UT the EIS slit was cutting across the erupting prominence and the corresponding emission is mapped at columns located around

\footnotetext{
${ }^{2}$ Please notice that the He II $\lambda 256.32$ line is blended with other coronal lines such as the $\mathrm{Si} x \lambda 256.36, \mathrm{Fe} \times \lambda 256.39$, Fe XII $\lambda 256.41$ and Fe XIII $\lambda 256.42$ spectral lines. The intensities of these line have not yet removed in the intensity map shown in Fig. 5: work to deblend the He II line is in progress.
} 

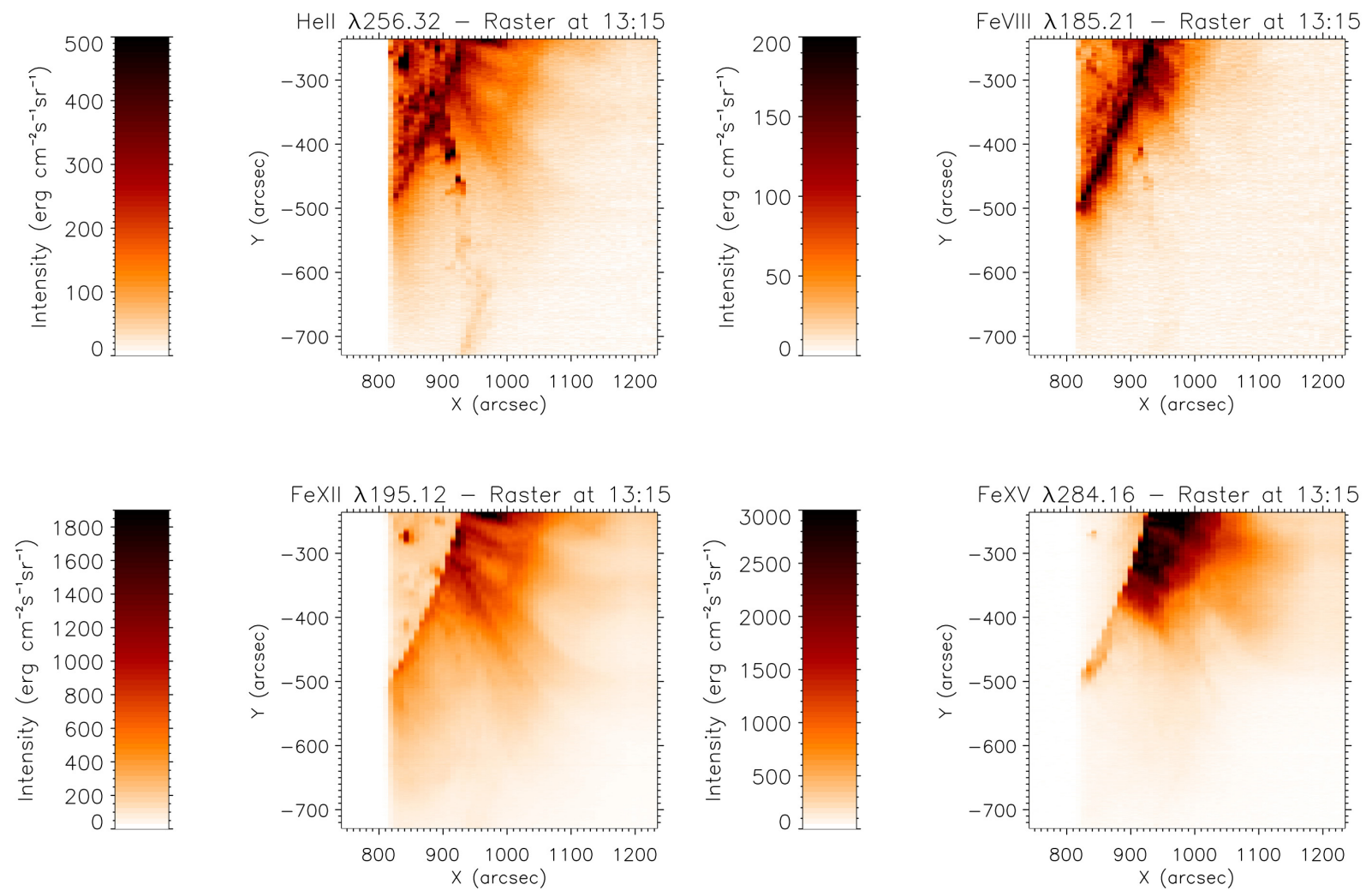

Fig. 5. The erupting filament material and the surrounding corona as seen by EIS in the He II $\lambda 256.32$ (top left), Fe VIII $\lambda 185.21$ (top right), Fe XII $\lambda 195.12$ (bottom left) and Fe XV $\lambda 284.16$ (bottom right) spectral line intensities during the 13:15-14:18 UT raster (see Fig. 4). The left part of the raster contains missing data.

$\mathrm{X} \simeq 950^{\prime \prime}$, while 10 min later $(\sim 14: 00 \mathrm{UT})$ the prominence is already out of the spectrometer FOV and the EIS slit is sampling the prominence remnants located at the projected position of the coronal region from where the eruption appears to expand. The EIS line intensity maps in the "hotter" lines such as Fe XII $\lambda 195.12$ (bottom left panel) and Fe XV $\lambda 284.16$ (bottom right) show no emission from the prominence material. These lines have higher maximum formation temperatures ( $T \sim 10^{6.2} \mathrm{~K}$ and $T \sim 10^{6.4} \mathrm{~K}$, respectively) hence this implies that the erupting plasma is at most a temperature of $10^{5.6} \mathrm{~K} \simeq 4 \times 10^{5} \mathrm{~K}$ (i.e. the Fe VIII maximum formation temperature).

Figure 5 shows that the high temperature emission is apparently not affected by the transit of the erupting filament. Nevertheless, strong changes in the high temperature line profiles occur: in particular, at projected positions where the erupting material is sampled all the observed coronal spectral lines show strong line broadenings. As an example, two Fe XII $\lambda 195.12$ normalized line profiles are plotted in Fig. 6 (top left panel): these two profiles have been acquired in the surrounding undisturbed corona (red line) and along the eruption path (blue line) at the same heliocentric distance. From a comparison between the two profiles it is evident that a strong line broadening is associated with the region where the prominence eruption occurred. Because, as shown by the bottom left panel of Fig. 5, EIS data show no significative emission from the erupting material in the Fe XII line, this non-thermal broadening has to be ascribed entirely to the surrounding corona. In order to better show this line broadening, Fig. 6 also shows the normalized Fe XII $\lambda 195.12$ line profiles along a row (top right) and along a column (bottom left) both cutting across the region of line profile broadening. In order to better show real broadenings before any data reduction, profiles shown here are extracted from raw data, hence before correcting for instrumental effects. For this reason, profiles plotted along a column (bottom left panel), i.e. along the slit, show a progressive centroid shift caused by the tilt between the spectrometer slit, the grating grids and the detector columns, while those plotted along a row (top right panel), i.e. at different times, show a quasi-sinusoidal line shift due to the HINODE spacecraft orbital motion occurring during the raster. From these two panels it is evident that strong line broadenings occur approximately in the FOV region between $880^{\prime \prime}<\mathrm{X}<980^{\prime \prime}$ and $\mathrm{Y}<-560^{\prime \prime}$.

The observed line profile widths are much larger than what expected from the theoretical thermal broadening (due to the 

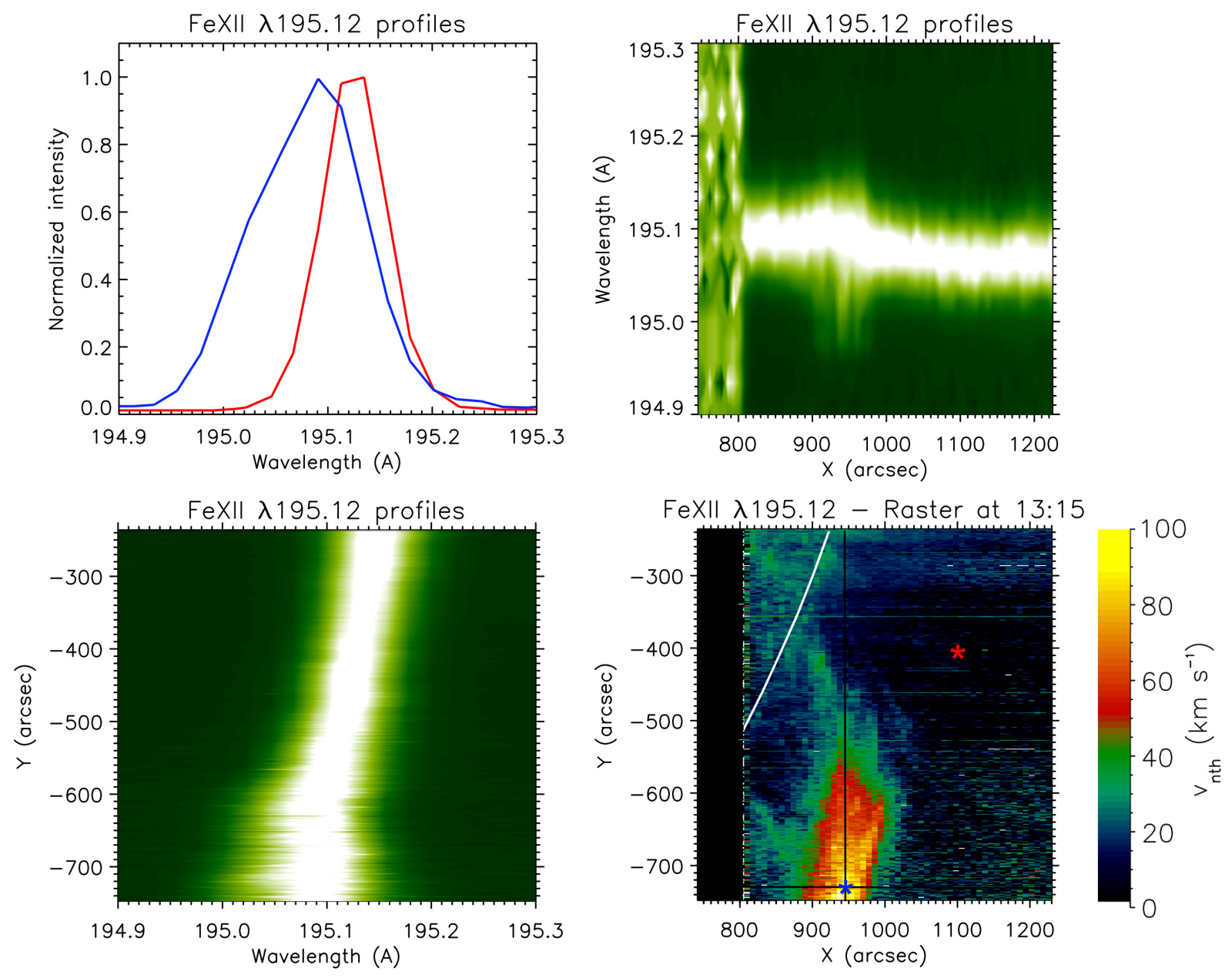

Fig. 6. Top left: a comparison between two Fe XII $\lambda 195.12$ line profiles acquired along the eruption path (blue line) and in the surrounding corona (red line). The corresponding positions where these two profiles have been acquired are outlined in the bottom right panel of this figure by a blue and a red asterisk, respectively. Top right and bottom left: normalized Fe XII $\lambda 195.12$ line profiles plotted along a detector row (top right) and a column (bottom left) cutting across the coronal region crossed by the prominence eruption (profiles shown in these panels are not corrected for the EIS slit tilt and the HINODE spacecraft motion; see text). Bottom right: a map showing the Fe XII $\lambda 195.12$ non thermal line broadening in the raster acquired immediately after the eruption (see Fig. 4 and bottom left panel in Fig. 5). Vertical and horizontal black lines show the detector column and row relative to the profiles plotted in the top right and bottom left panels.

ion kinetic temperature) and the instrumental broadening. Hence, in order to estimate the amplitude of the observed non-thermal broadenings, it is necessary to remove from FWHMs estimated with gaussian fitting the broadenings due to these two effects. The instrumental FWHM for the $2^{\prime \prime}$ slit is $\simeq 3.0$ pix $=0.0669 \AA$, while the thermal broadening for the Fe XII ion (with temperature of maximum formation of $10^{6.2} \mathrm{~K}$ ) is $0.0237 \AA$, hence the expected line profile FWHM should be on the order of $\sqrt{0.0669^{2}+0.0237^{2}} \AA=0.071 \AA$ in good agreement with the profiles observed in the unperturbed corona (red profile in the top left panel of Fig. 6). Once the instrumental and thermal broadenings are subtracted, it turns out that the Fe XII $\lambda 195.12$ line has along the eruption path strong non-thermal line broadenings, up to $\simeq 0.1 \AA$, corre- sponding to non thermal velocities up to $\sim 100 \mathrm{~km} \mathrm{~s}^{-1}$ (i.e. a kinetic temperature $\simeq 3.5 \times 10^{7} \mathrm{~K}$, far in excess of the ion kinetic temperature of $1.6 \times 10^{6} \mathrm{~K}$ ). In order to better show this effect, a map of Fe XII $\lambda 195.12$ non-thermal line broadenings is shown in Fig. 6 (bottom right panel): by comparing this map with Fig. 5 (top left panel) and with the evolution shown in Fig. 4 it becomes evident that the observed non-thermal broadenings are located in the coronal region crossed by the erupting prominence. Interestingly, line fitting to spectral lines from higher $\mathrm{Fe}$ ionization stages (such as Fe XIII $\lambda 202.04$, Fe XIV $\lambda 274.20$ and Fe XV $\lambda 284.16$ ) indicates that this effect progressively decreases for increasing Fe ionization stages. For instance, the non-thermal broadening observed in the Fe XV $\lambda 284.16$ line are approximately 

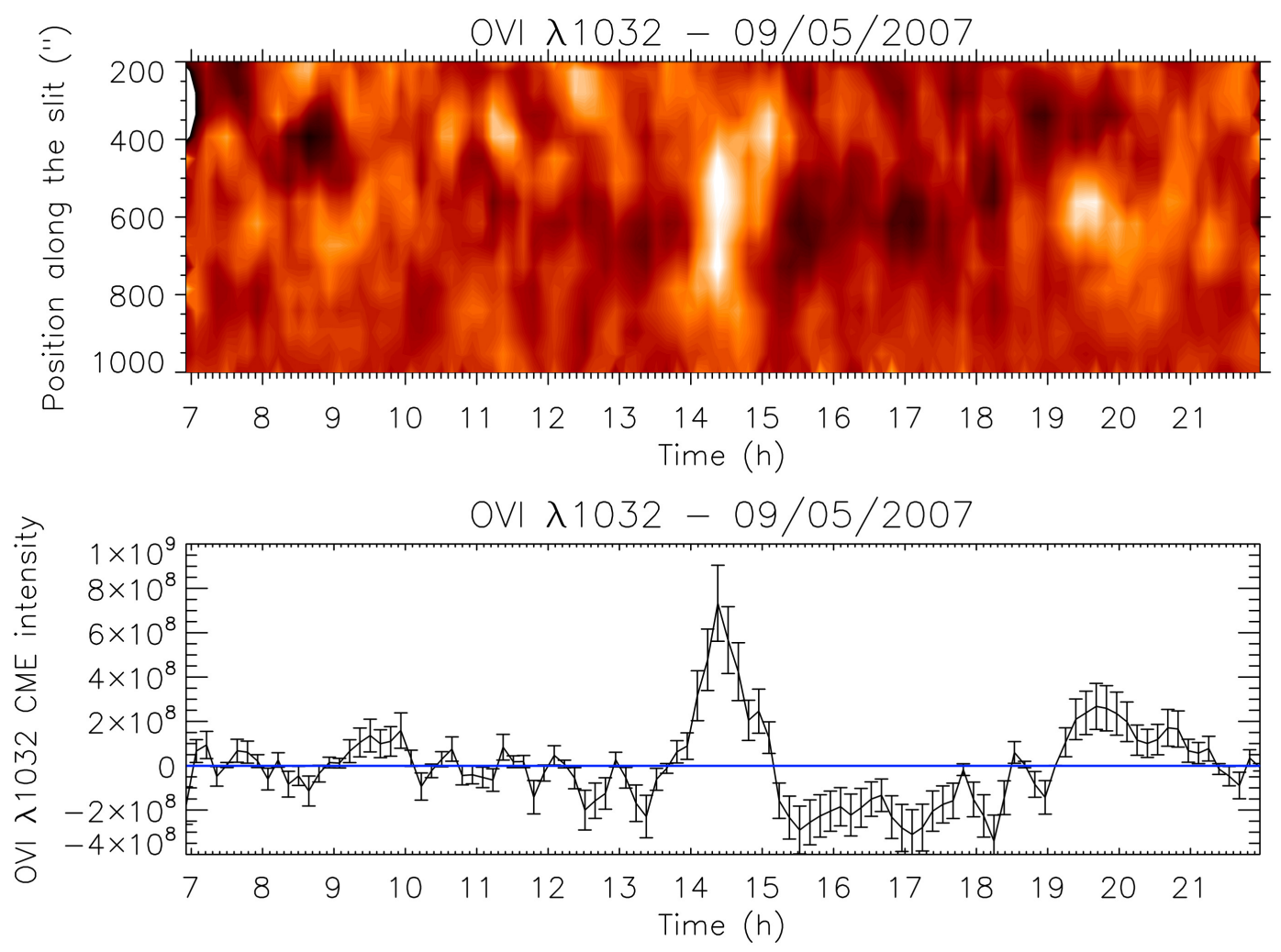

Fig. 7. Top: the evolution of the O VI $\lambda 1031.9$ line intensity along the southward part of the UVCS slit as a function of time. Positions along the slit (y-axis) are given in arcsecs measured from the slit center; the pre-CME average coronal intensity has been subtracted in order to enhance the visibility O VI line intensity increase occurring between $~ 14: 00$ and 15:10 UT and due the CME transit across the slit. Bottom: the O VI $\lambda 1031.9$ line intensity evolution averaged over the southward part of the UVCS slit shown in the top plot (average preCME intensity subtracted). The intensity peak between 14:00 and 15:10 UT corresponds to the transit of the CME front, while the following intensity decrease can be interpreted as the CME void.

$1 / 2$ of the Fe XII $\lambda 195.12$ line broadenings observed in the same location (a detailed analysis of the instrumental width is in progress, together with an assessment of blending in the EIS lines). Possible explanations for these broadenings are discussed in Sect. 5.

\section{SOHO/UVCS observations}

In the higher corona the eruption reported here has been observed by the SOHO/UVCS spectrometer; this instrument is able to observe spectral lines in two wavelength bands (Ly$\alpha$ channel: $1145-1287 \AA$; O VI channel: $937-1126 \AA$ ) with a spectral resolution of $0.14 \AA /$ pixel and $0.0925 \AA /$ pixel respectively and a spatial resolution of $7^{\prime \prime}$; the projected slit FOV, always oriented perpendicular to the radial direction, is $40^{\prime}$ long and its thickness can be varied from $\sim 14^{\prime \prime}$ to $\sim 83^{\prime \prime}$. On 9 May 2007 the UVCS instrument acquired spectra with the slit centered at an heliocentric distance of $1.7 \mathrm{R}_{\odot}$ and a latitude of $5^{\circ} \mathrm{SW}$ (see Fig. 3). The slit thus covered, with its southernmost section, also the region where the filament expansion and CME have been observed, and the parame- ters derived from its data represent the physical condition of plasma above the area sampled by HINODE experiments.

In particular, during the CME transit UVCS data show a relatively faint $(\sim 20 \%)$ increase in the O VI $\lambda \lambda 1031.9-$ 1037.6 doublet lines (see Fig. 7) and a smaller increase also in the Si XII $\lambda 520.6$ line (second order line). This intensity increase starts around 14:00 UT, when the erupting prominence was located at lower altitudes (see Fig. 1, top right panel): this led us to conclude that the observed jump in the $\mathrm{O}$ VI intensity represents the CME front formed by the coronal plasma compressed by the expanding prominence located at lower levels; the intensity peak is also followed by an intensity decrease which can be associated to the CME void, while we did not expect to observe significative emission from the prominence. In fact, because the $\mathrm{O}$ VI line has a temperature of maximum formation around $3.2 \times 10^{5} \mathrm{~K}$, the cooler chromospheric prominence plasma (whose temperature decreases with time as the prominence adiabatically expands) is usually not emitting in this line, explaining why the transit of the prominence is not observed by UVCS in the $\mathrm{O}$ VI spectral lines. 
As well known, in the solar corona, lines form both by excitation due to the collisions with thermal electrons (collisional excitation) and by absorption of radiation emitted by the underlying layers (radiative excitation). Emission in the Si XII line is due only to collisional excitation, while lines from neutral Hydrogen and from the O VI ion form also by radiative excitation. The theoretical ratio between the intensities of the collisional components of the O VI $\lambda 1031.9$ and $\lambda 1037.63 \AA$ lines is 2.0 while the ratio between their radiative components is 4.0 (see discussion in Noci et al., 1987). Because the average ratio observed in the pre-CME corona is 2.5 , this implies that $60 \%$ of the 1031.9 line is collisionally excited while $40 \%$ of the line is made of photons scattered from the solar disk. Assuming the pre-CME plasma to be stationary, its electron density $n_{e}$ can be derived from the ratio of the collisional to the radiative line components. For a coronal electron temperature $T_{e}=10^{6.3} \mathrm{~K}$ (as estimated from the ratio of the $\mathrm{O}$ VI $\lambda 1031.9$ collisional component to the Si XII $\lambda 520.6$ line intensity) and by assuming an exciting disk intensity $I_{\text {disk }}=1.94 \times 10^{13}$ phot $\mathrm{cm}^{-2} \mathrm{~s}^{-1} \mathrm{sr}^{-1}$ (as measured by UVCS in 1996 at about the same phase of the solar cycle; see Raymond et al., 1997) we infer an electron density of the pre-CME corona $n_{e}=7 \times 10^{6} \mathrm{~cm}^{-3}$. The same technique applied to the post-CME corona gives approximately the same temperature, while the coronal density decreases by about $15 \%$; this can be expected if the passage of the CME partially evacuated the corona.

The average density of the CME front can be estimated as follows: the CME is moving at $\sim 310 \mathrm{~km} \mathrm{~s}^{-1}$, hence the $\mathrm{O} \mathrm{VI}$ radiative component will be completely Doppler dimmed. The ratio between the O VI $\lambda 1031.9$ and $\lambda 1037.63 \AA$ lines observed at the CME (i.e. after the subtraction of the pre-CME intensity) is $\sim 2$, hence pumping of the $\mathrm{O}$ VI $\lambda 1031.9$ line radiative component by the $\mathrm{C}$ II $\lambda 1037.0$ and $\mathrm{C}$ II $\lambda 1036.3$ spectral lines can be ruled out for the present event and the observed O VI intensity increase can be ascribed solely to the increase in the collisionally excited component of this line (see Kohl et al., 1997, for a discussion of the Doppler dimming effect in the $\mathrm{O}$ VI lines). The latter is roughtly proportional to the quantity $\propto n_{e}^{2} L$, where $L$ is the extension along the LOS of the emitting plasma. By assuming that the front is cilindrically symmetric, hence that $L$ equals the observed extension along the UVCS slit $\left(0.65 \mathrm{R}_{\odot}\right.$, see Fig. 7 , top panel), it turns out that the transit of the CME front corresponds to a density increase by $3 \times 10^{6} \mathrm{~cm}^{-3}$ with respect to the preCME corona: hence, the CME front, formed by compression of the coronal plasma, is denser by more than $40 \%$ than the surrounding corona.

\section{Conclusions}

We presented here results from the analysis of a unique data set which allowed us to study a prominence eruption (resulting in a slow CME) observed from STEREO/EUVI, HIN-
ODE/EIS and SOHO/UVCS data on 9 May 2007. Data acquired by the EUVI telescopes onboard STEREO-A and -B spacecraft have been used here to derive information on the 3-D structure of the erupting prominence. Observations acquired from these instruments have already been used to infer the 3-D shape of coronal loops (Feng et al., 2007; Aschwanden et al., 2008; Rodriguez et al., 2009) and polar plumes (Curdt et al., 2008). Recently Mierla et al. (2008) estimated the propagation direction of CMEs by using the leading edges height-time curves measured from STEREO COR-1 A \& B images, while Howard and Tappin (2008) inferred by triangulation the position of the source region of two CMEs. Gissot et al. (2008) developed an algorithm to reconstruct from a pair of EUVI images a map of heights above the solar surface across an erupting filament. An erupting prominence has been also studied in 3-D by Thompson (2008) who found a prominence rotation by $\sim 140^{\circ}$ in the rising phase. However, only a few events have been studied so far in 3-D in their early erupting phase and the global picture of CME development is far from being completely understood. The information we derived in this work from STEREO on the 3-D structure of the erupting filament led us to conclude that the prominence is a "hook-shaped" mainly 2-D planar structure, suggesting the absence of a 3-D flux rope, even if this kind of structures is envisaged in many flare-CME models. The triangulation study has been performed $\sim 12 \mathrm{~min}$ after the eruption has been triggered, hence we cannot exclude that twisting motions occurred at earlier times. The prominence material rises at larger altitudes and also moves at higher latitudes (showing a southward motion), while no significant motion occurs in the longitudinal direction, as demonstrated by the North-South orientation of the prominence and by the coincidence of the average prominence material longitude with that of the source Active Region. This suggests that the prominence expansion occurs mainly over a plane, hence is anysotropic. Unfortunately, at the time of the eruption the source AR was already $14^{\circ}$ behind the limb, hence no information are available for this event on the triggering mechanisms.

The prominence eruption has been observed by the HINODE/EIS spectrometer during an off-limb West-East spatial raster. The spectra acquired by EIS showed unexpected non-thermal velocities in the corona along the eruption path, up to $\sim 100 \mathrm{~km} \mathrm{~s}^{-1}$. Interestingly, non-thermal broadenings are observed in high temperature spectral lines, such as the Fe XII $\lambda 195.12$ line, whose intensity maps show no emission in areas where the cool chromospheric material erupted within the prominence. This implies that the observed broadenings are related to a phenomenon occurring not in the prominence plasma, but in the corona crossed by the prominence and/or surrounding the eruption site and aligned along the LOS. These broadenings could be in principle ascribed to many phenomena such as: 1) plasma heating, due to a) magnetic reconnections occurring during or after the eruption, $b$ ) adiabatic compression due to the prominence expansion, or 
c) other physical processes, 2) plasma turbulences (induced by reconnection), 3) fan shaped outflows projecting at different angles with respect to the LOS (along field lines opened by the CME), or other effects. Nevertheless, the observed broadening dependence on the Fe charge state suggests to rule out explanations involving collective plasma motions (such as plasma turbulence and outflows) and to favor charge dependent heating processes (such as the adiabatic compression). A mechanism dependent on the ion charge or on its mass-to-charge ratio is playing a role: this could be for instance the magnetic reconnection (as recently indicated by laboratory plasma experiments; see Gangadhara et al., 2008), but other phenomena are possible (as the ion-cyclotron resonance; see e.g. Cranmer et al., 1999). Data analysis and interpretation are still in progress, hence these possibilities will be further investigated in a future work. We notice that similar post-eruption non thermal broadenings have been recently reported by McIntosh (2009) and, based on the association with EUV dimmings, interpreted as the effect of Alfvén waves propagating along the magnetic fieldlines left open by the CME. Nevertheless, opposite to our case, these broadenings were observed after the eruption and the association with coronal dimming has not yet been investigated in the present work.

The CME associated with the prominence eruption and visible in white light coronagraphs has been observed by the SOHO/UVCS spectrometer in the lower corona. This is not a large scale event, and the variations observed in the UVCS specral line intensities are small. Nevertheless, a relatively faint $(\sim 20 \%)$ increase in the O VI $\lambda \lambda 1031.9-1037.6$ doublet lines and a smaller increase in the Si XII $\lambda 520.6$ line are observed. At $\sim 1.7 \mathrm{R}_{\odot}$ these increases are observed to start around 14:00 UT, when the prominence was still in the FOV of the STEREO/EUVI telescope: this led us to conclude that the $\mathrm{O}$ VI intensity variations are due to the transit of the CME front, located above the erupting prominence and formed by compression of the overlying coronal plasma. As we derived from the O VI and Si XII line intensities, this compression increases the local density by $\sim 40 \%$ from $7 \times 10^{6} \mathrm{~cm}^{-3}$ up to $1 \times 10^{7} \mathrm{~cm}^{-3}$. There are not many density estimates in mass ejections: Akmal et al. (2001) found densities $>7.10^{8} \mathrm{~cm}^{-3}$ at $1.3 \mathrm{R}_{\odot}$, Raymond and Ciaravella (2004) at $3 \mathrm{R}_{\odot}$ give values ranging between $1.3 \times 10^{6}$ and $4 \times 10^{7} \mathrm{~cm}^{-3}$, Bemporad et al. (2007) at $1.6 \mathrm{R}_{\odot}$ estimated densities between $7.4 \times 10^{6}$ and $1.1 \times 10^{7} \mathrm{~cm}^{-3}$ depending on whether densities referred to the core or the void of the CME. We conclude that present densities are consistent with previous estimates.

Even if the data analysis and interpretation are still in progress, this initial study demonstrates the great potentiality of STEREO, HINODE and SOHO multi-spacecraft campaigns for the study of solar eruptions.

Acknowledgements. A.B. and M.M. acknowledge support from ASI/INAF I/035/05/0 contract. SOHO is a Mission of international cooperation between ESA and NASA. STEREO is a NASA mis- sion. Hinode is a Japanese mission developed and launched by ISAS/JAXA, with NAOJ as domestic partner and NASA and STFC (UK) as international partners. It is operated by these agencies in co-operation with ESA and NSC (Norway).

Topical Editor R. Forsyth thanks B. Schmieder and another anonymous referee for their help in evaluating this paper.

\section{References}

Akmal, A., Raymond, J. C., Vourlidas, A., et al.: SOHO Observations of a Coronal Mass Ejection, Astrophys. J., 553, 922-934, 2001.

Aschwanden, M. J., Wülser, J.-P., Nitta, N. V., and Lemen, J. R.: First Three-Dimensional Reconstructions of Coronal Loops with the STEREO A and B Spacecraft. I. Geometry, Astrophys. J., 679, 827-842, 2008.

Bemporad, A., Del Zanna, G., Andretta, V., et al.: An erupting filament and associated CME observed by Hinode, STEREO and SOHO, Proc. of the "2nd HINODE Science Meeting", Boulder (CO), in press, 2009a.

Bemporad, A.: Stereoscopic Reconstruction from STEREO/EUV Imagers Data of the Three-dimensional Shape and Expansion of an Erupting Prominence, Astrophys. J., 701, 298-305, 2009 b.

Bemporad, A., Raymond, J. C., Poletto, G., and Romoli, M.: A Comprehensive Study of the Initiation and Early Evolution of a Coronal Mass Ejection from Ultraviolet and White-Light Data, Astrophys. J., 655, 576-590, 2007.

Cranmer, S. R., Field, G. B., and Kohl, J. L.: Spectroscopic Constraints on Models of Ion-cyclotron Resonance Heating in the Polar Solar Corona, Space Sci. Rev., 87, 149-152, 1999.

Culhane, J. L., Harra, L. K., James, A. M., et al.: The EUV Imaging Spectrometer for Hinode, Solar Phys., 243, 19-61, 2007.

Curdt, W., Wilhelm, K., Feng, L., and Kamio, S.: Multi-spacecraft observations of polar coronal plumes, Astron. Astrophys., 481, L61-L64, 2008.

Del Zanna, G., Andretta, V., Poletto, G., et al.: Multi-instrument campaigns to observe the off-limb corona, Proc. of the " 2 nd HINODE Science Meeting”, Boulder (CO), in press, 2009.

Feng, L., Inhester, B., Solanki, S., et al.: First Stereoscopic Coronal Loop Reconstructions from STEREO SECCHI Images, Astrophys. J., 671, L205-L208, 2007.

Gangadhara, S., Craig, D., Ennis, D. A., et al.: Ion heating during reconnection in the Madison Symmetric Torus reversed field pinch, Phys. Plasmas, 15, 056121-056121-9, 2008.

Gissot, S. F., Hochedez, J.-F., Chainais, P., and Antoine, J.-P.: 3D Reconstruction from SECCHI-EUVI Images Using an OpticalFlow Algorithm: Method Description and Observation of an Erupting Filament, Solar Phys., 252, 397-408, 2008.

Kohl, J. L., Esser, R., Gardner, L. D., et al.: The Ultraviolet Coronagraph Spectrometer for the Solar and Heliospheric Observatory, Solar Phys., 162, 313-356, 1995.

Kohl, J. L., Noci, G., Antonucci, E., et al.: First Results from the SOHO Ultraviolet Coronagraph Spectrometer, Solar Phys., 175, 613-644, 1997.

Mierla, M., Davila, J., Thompson, W., et al.: A Quick Method for Estimating the Propagation Direction of Coronal Mass Ejections Using STEREO-COR1 Images, Solar Phys., 252, 385-396, 2008. 
McIntosh, S. W.: The Inconvenient Truth About Coronal Dimmings, Astrophys. J., 693, 1306-1309, 2009.

Noci, G., Kohl, J. L., and Withbroe, G. L.: Solar wind diagnostics from Doppler-enhanced scattering, Astrophys. J., 315, 706-715, 1987.

Raymond, J. C. and Ciaravella, A.: Densities and Velocities in Fast Coronal Mass Ejections: Radiative Pumping of the O VI Doublet, Astrophys. J., 606, L159-L162, 2004.

Raymond, J. C., Kohl, J. L., Noci, G., et al.: Composition of Coronal Streamers from the SOHO Ultraviolet Coronagraph Spectrometer, Solar Phys., 175, 645-665, 1997.

Rodriguez, L., Zhukov, A. N., Gissot, S., and Mierla, M.: ThreeDimensional Reconstruction of Active Regions, Solar Phys., 256, 41-55, 2009.
Howard, T. A. and Tappin, S. J.: Three-Dimensional Reconstruction of Two Solar Coronal Mass Ejections Using the STEREO Spacecraft, Solar Phys., 252, 373-383, 2008.

Thompson, W. T.: Coordinate systems for solar image data, Astron. and Astrophys., 449, 791-803, 2006.

Thompson, W. T.: 3D Reconstruction of an Erupting Prominence, Proc. of the "AGU Fall Meeting 2008", abstract n. SH13B-1520, 2008.

Wuelser, J.-P., Lemen, J. R., Tarbell, T. D., et al.: EUVI: the STEREO-SECCHI extreme ultraviolet imager, Proceedings of the SPIE, 5171, 111-122, 2004. 